Conference Proceedings - Long Paper

\title{
It Takes a 'Micro-Village': A new understanding of the relationship between socio-spatial infrastructure and equitable resilience
}

\author{
Jess Pauly \\ Universitat Internacional de Catalunya (UIC) / Carrer de la Immaculada, 22, 08017 Barcelona \\ E-Mail: jesspauly@gmail.com
}

\begin{abstract}
:
Humanity's values and priorities are reflected in the spatial organization of the environments we create and occupy. Yet ecological instability and societal inequity, intrinsic to the growth-prioritized societal model, demand an urgent shift in socio-spatial organization. In understanding that actionable investment is contingent not only on scientific consensus and political will, but also on psychosocial culture, this research endeavors to communicate the importance of linguistic and visual framing as crucial tactics capable of influencing culture away from a growth-prioritized organization, and towards a model which comprehends needs as an ecosystem of interdependent variables; an understanding necessary to effectively construct a more resilient paradigm of living. In order to ensure this new paradigm will facilitate equity, resilience must be understood from the bottom-up and assessed as Personal Operating Power [POP].
\end{abstract}

Based on these three understandings, this research proposes the following multi-scalar linguistic framing as a means of reconceiving our socio-spatial environments in an ecosystemic manner:

'Micro-Village': denotes the ecosystem at the personal / domicile scale

'Macro-Village': denotes an ecosystem at the community / regional scale; a network of Micro-Villages, typically with spatial continuity

'Multi-Village': denotes an ecosystem of networked Macro-Villages 
In addition, visual, diagrammatic communication has been developed based on insights from those subjugated under the growth-prioritized model, in an effort to facilitate comprehension of how one's socio-spatial context intersects with a healthy ecosystem of needs, and can be used to facilitate bottom-up resilience, understood as POP.

This research strives to bridge the comprehension gap between scientific academia, community organizers, and the general public, providing tools of communication to help facilitate new framing strategies necessary to shift humanity's socio-spatial organization to a model which promotes both ecological stability and societal equity.

Keywords: Equitable Resilience; Personal Operating Power [POP]; Ecosystem of Needs; Socio-spatial Infrastructure; Micro-Village; Macro-Village; Multi-Village.

\section{Introduction}

Ecological instability and societal inequity clearly demonstrate the inadequacy of the growth-based model, commonly known as capitalism, and establish an indisputable need for systemic change in societal organization. Yet at current, two simultaneous forces are at play-the need for large-scale, globalized investment in order to shift the globalized societal infrastructure from one of growth to one of ecosystemic prioritization, as well as bottom-up control in decision-making, to subvert existing hierarchical abuses of power which have exacerbated both injustice and ecological instability.

\section{Current State}

The 2018 UN Intergovernmental Panel on Climate Change (IPCC) report comprehensively illustrates the absence of ecosystemic resilience and an immediate need to shift away from the current growth-based societal system. Yet, even if the reality of these ecological consequences were not considered, the hierarchical structural inequity of access to the fulfillment of human needs shown to be perpetual in this model- despite overwhelming wealth and capacity of production- simultaneously demands this systemic shift away from growth as well (Piketty 2004; Chomsky 2013; Baradaran 2017).

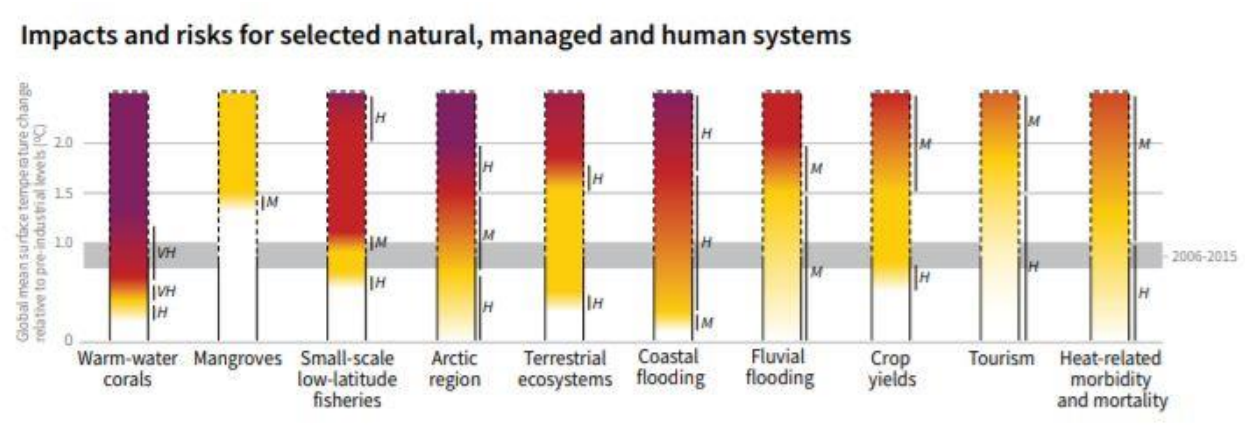

Confidence level for transition: L=Low, M=Medium, H=High and VH=Very high

Figure 1. Source: IPCC Report 2018 
Sea level rise threatens to displace millions from their homes within the next few decades ${ }^{1}$, including over 380,000 households in the $\mathrm{US}^{2}$, where skyrocketing rental costs from market-dominated housing is increasing displacement via speculation and gentrification, increasing housing insecurity and homelessness (Gee 2017). Industrial monoculture agriculture increases vulnerability to climate change impacts such as increasingly intense droughts and heatwaves, as well as crop loss due to disease and storms of increasing severity ${ }^{3}$. Dependency on fossil fuels and large-scale monopolydominated electrical networks greatly exacerbates ecological instability, environmental contamination, and the vulnerability of disenfranchised communities, particularly in the aftermath of large-scale storms (Klein 2018) which will only increase in intensity and frequency as sea temperatures rise. A lack of access to fresh produce and local grocery stores- commonly termed 'food deserts' but more accurately described by food justice advocate Karen Washington as food apartheid ${ }^{4}$ - has had a range of implications for infrastructurally marginalized neighborhoods (Anguelovski 2015), including a reemergence and upsurge of scurvy in the United States, due to a rise in nutritional deficiencies ${ }^{5}$. And, crucially, as expressed in Figure 2, all of these consequences intersect with and affect individual health and psychological well-being.

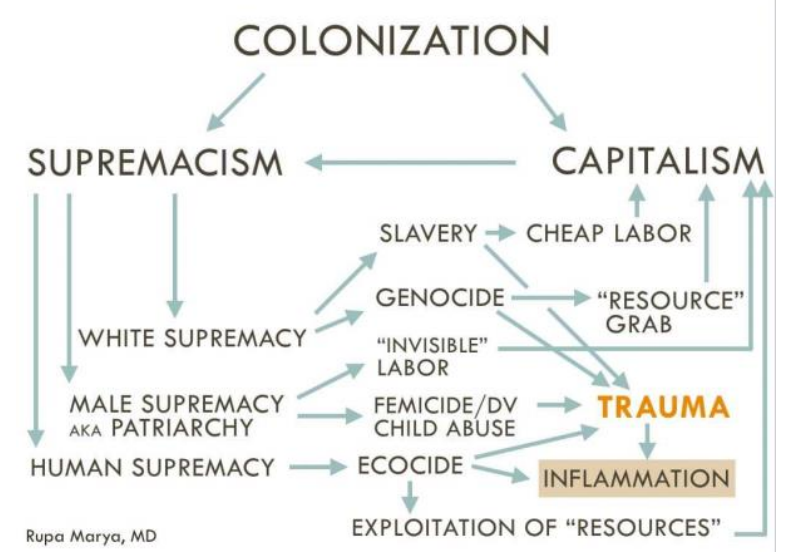

Figure 2. Graphic created by physician Rupa Marya M.D.; keynote presentation at the 2018 Bioneers Conference, San Rafael, CA

With the release of the 2018 United Nations IPCC report, consensus has confirmed what manyeven the Pope ${ }^{6}$ - have urgently sought to warn - the consequences of the existing growth-based model of societal organization have impacted the biosphere at a rate of change that is causing ecological instability which will result in a multitude of ongoing, devastating consequences for both human society and the natural environment, and much sooner than social society comprehends. Anthropologist Jason Hickel explains that "even if we do everything right, decoupling economic growth with resource use will remain elusive and our environmental problems will continue to worsen. Preventing that outcome will require a whole new paradigm. [...] Ultimately, bringing our civilization back within planetary boundaries is going to require that we liberate ourselves from our dependence on economic growth—starting with rich nations" (Hickel 2018).

\footnotetext{
${ }^{1}$ David Wallace-Wells, "UN Says Climate Genocide Is Coming. It's Actually Worse Than That."

${ }^{2}$ NOAA, "Patterns and Projections of High Tide Flooding along the U.S. Coastline Using a Common Impact Threshold."

${ }^{3}$ Robert McSweeney and Rosamund Pearce, "Interactive: Carbon Brief"

${ }^{4}$ Anna Brones, "Food Apartheid: The Root of the Problem with America's Groceries."

${ }^{5}$ Leah Samuel, "Not Just Pirates Anymore: Scurvy Afflicts the Poor and Homeless."

${ }^{6}$ Naomi Klein, “A Radical Vatican?"
} 
In similar sentiment prior to a post-growth conference held in September of 2018, a coalition of 238 academics signed an open letter toward policymakers declaring that "We are now exceeding the safe operating space for humanity on this planet, and there is no sign that economic activity is being decoupled from resource use or pollution at anything like the scale required. [...] Right now the response is to try to fuel growth by issuing more debt, shredding environmental regulations, extending working hours, and cutting social protections. This aggressive pursuit of growth at all costs divides society, creates economic instability, and undermines democracy"7.

The coalition of academics then made four demands of policymakers, with the third being of crucial note:

\section{Turn the stability and growth pact (SGP) into a stability and wellbeing pact. The SGP is a set of rules aimed at limiting government deficits and national debt. It should be revised to ensure member states meet the basic needs of their citizens, while reducing resource use and waste emissions to a sustainable level.}

This demand critically articulates the imperative need to shift from a growth-prioritized to ecosystemic paradigm of socio-spatial organization, one which prioritizes the fulfillment of needs and well-being, for both humans and the biosphere. Shifting paradigms will require a corresponding shift in cultural psychology and valuation of needs.

\section{Methodology}

\subsection{Linguistic and Visual Framing as Psychosocial Societal Infrastructure}

It does not feel unusual to observe an individual seemingly act against their own well-being. Yet while an outside observer may view an individual's actions as illogical, there is still an internal narrative of logic driving the individual's motivations that the outside observer is not privy to (Lakoff 1996). Cognitive linguist George Lakoff argues that this internal logic reflects an individual's psychosocial framework. To effectively communicate with those who hold a different cognitive logic, Lakoff suggest that attention to intention in linguistic communication is essential, and that linguistic framing functions as a form of psycho-social infrastructure which can either reinforce or change a given perspective or comprehension (Lakoff 2004).

Examples of growth-based linguistic framing include the categorical dichotomies of 'formal' vs. 'informal', 'legal' vs. 'illegal', and 'developed' vs. 'developing', among many others. All of these terms are framed with a value-based judgement, which perpetuates the normalization of Global North culture, the growth-prioritized power hierarchy, and capitalist infrastructure.

\footnotetext{
${ }^{7}$ Letters, "The EU Needs a Stability and Wellbeing Pact, Not More Growth."
} 
Similarly, visual framing can influence comprehension of data. An example of how the chosen parameters of a graphic can influence perception can be seen in the New York Times November 2018 post-election results coverage. Fig. 3 (left) portrays the electoral outcome in terms of land area, while the pictogram in Fig. 4 (right) uses population as the secondary parameter. If an individual were only informed by the image on the left, a perception of conservative dominance would seem logical. When population density is accounted for, the pictogram more accurately reflects the results, and it becomes clear how accurate comprehension of data is contingent on the representational parameters chosen.

\section{Figures 3 - 4}
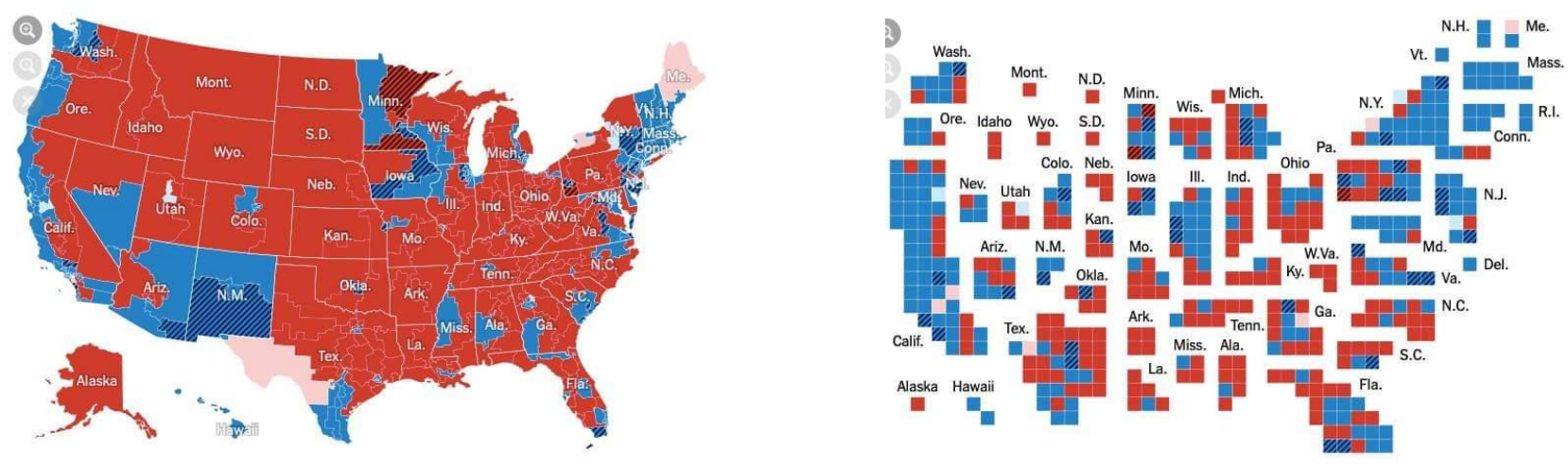

\subsection{Reframing Needs: from 'Hierarchy' to 'Ecosystem'}

The linguistic framing of needs as a 'hierarchy', originally conceived of by Abraham Maslow in his 1943 "Theory of Human Motivation", suggests an internalized understanding of human needs as having a relative hierarchy of importance. The original interpretation of Maslow's theory dictated that lower levels within the hierarchy were prerequisite to the pursuit or attainment of the needs above. This progressive, sequential growth closely mirrors the international community's understanding of 'development', and is reflected in the labeling of 'developed' and 'developing' nations which reinforces the growth-based psychology of liberal capitalism. While there is validity in articulating the differences in temporal urgency of needs, the linguistic framing of 'hierarchy of needs' has transferred to reinforce the psychology of global management; a management system which has perpetuated societal inequity, and ecological imbalance derived from the maintenance of the societal power hierarchy.

To adequately internalize the value of ecosystemic interdependence of needs, a new visual model is required to reflect the ecosystemic nature of needs. 

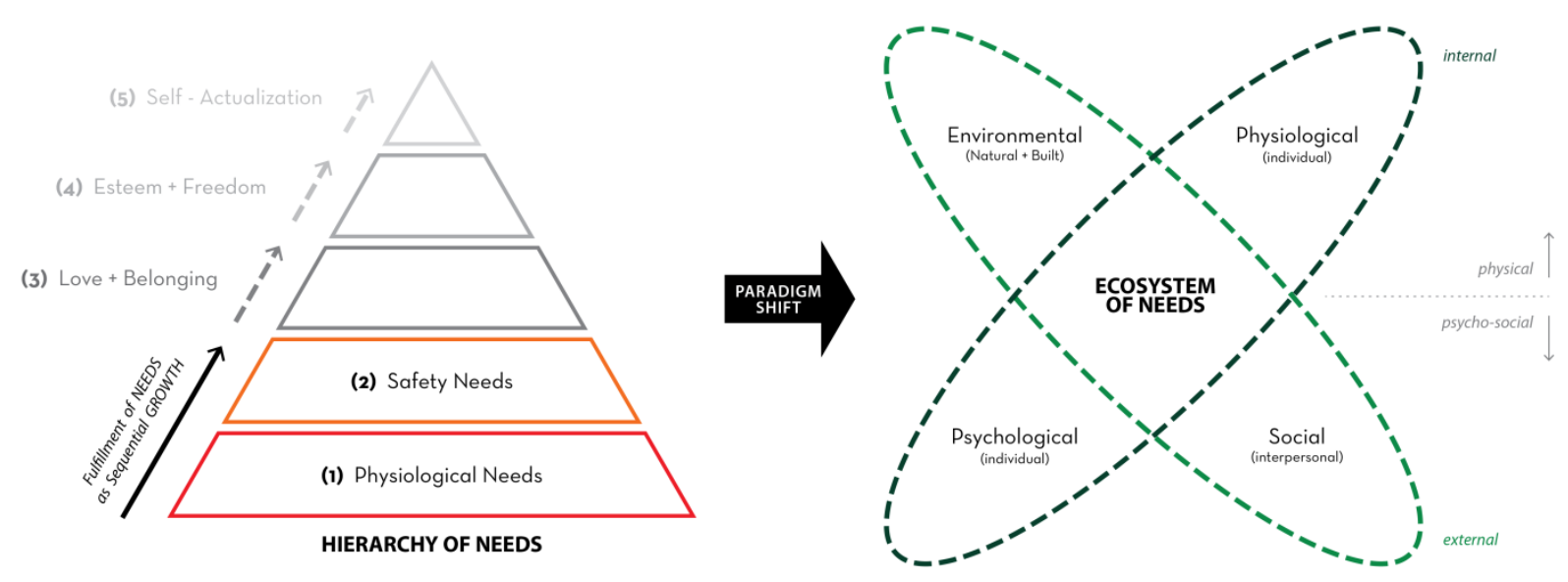

This new paradigm recognizes that internal needs, both physiological and psychological, are interdependent with one another. Simultaneously, these internal variables both affect and are affected by social influences of interpersonal interaction, as well as the physical environment- both built and natural. Where the old model infers that experiences such as respect and self-actualization are pursued upon the fulfillment of more "basic" needs -such as safety and food- this new framing proposes that well-being is predicated on a healthy ecosystem of inter-related, multi-scalar variables and their related needs. In this sense, a healthy ecosystem is the source of motivation, rather than the continual pursuit of 'development'.

\subsection{Understanding 'Resilience' as 'Personal Operating Power'}

In listening to systemically marginalized perspectives, both historic and contemporary, through an extensive literature review, informal interviews, and analysis of primary and secondary sources, a takeaway that becomes clear is that when it comes to prioritizing time and labor- physical and emotional- childcare, putting food on the table, and paying the energy bills will always take precedence because these are needs, while political and/or community organizing is considered a luxury. Yet within the current top-down system, political engagement is one of the only means for influencing large-scale public, economic investment; an investment, which is necessary to achieving the scale of change necessary for adequate ecological impact. When there are also additional barriers to political engagement such as voter disenfranchisement targeting already systemically marginalized communities, this instability in Personal Operating Power [POP] cyclically perpetuates the existing infrastructural power dynamic. Learning from this is critical and understanding how to transmit that comprehension to achieve critical mass in societal internalization is fundamentally important.

Typically, in community and regional resilience, there are three commonly understood traits: preparedness, diversity, and self-organization (Schipper and Langston 2015). At the individual scale, resilience should be understood as 'Personal Operating Power' [POP]. 
Figure 6. Image created by author.

Community Resilience

(1) Preparedness

(2) Diversity

(3) Self-Organization
Personal Operating Power

(1) Fulfillment of Needs

(2) Equity of Access

(3) Bottom-Up Sovereignty

Personal Operating Power [POP] refers to:

(1) Security of localized, flexible access to the fulfillment of the ecosystem of human needs

(2) Aforementioned access should include a range of readily available tactics which can accommodate and flexibly adapt to the range of context and individual experience

(3) There should be autonomy in deciding which tactics are most suitable for one's own ecosystem of needs, which can be understood as 'sovereignty'.

To empower POP, it becomes necessary to facilitate comprehension of the needs of the environment, individuals, and society as interdependent. Therefore, this ongoing body of research endeavors to propose linguistic and visual framing which promotes the cultural understanding and internalization of the ecosystemic model.

\section{Proposals}

\subsection{Proposal: Linguistic framing to facilitate comprehension and communication}

As both ecological instability and societal inequality must be addressed, the following terminology is proposed in order to psychologically link the vital interdependence between the ecosystem of needs and socio-spatial context-- individual, interpersonal, and environmental. Understanding socio-spatial environments as ecosystems can contribute to building resilience.

'Micro-Village': denotes the ecosystem at the personal / domicile scale

'Macro-Village': denotes an ecosystem at the community / regional scale; and/or a network of Micro-Villages, typically with spatial continuity

'Multi-Village': denotes an ecosystem of networked Macro-Villages 
Figure 7. Image created by author.

MICRO - Village

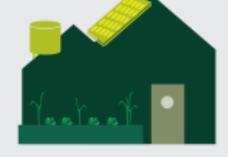

MACRO - Village

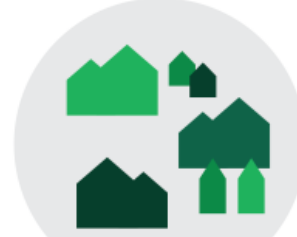

MULTI - Village
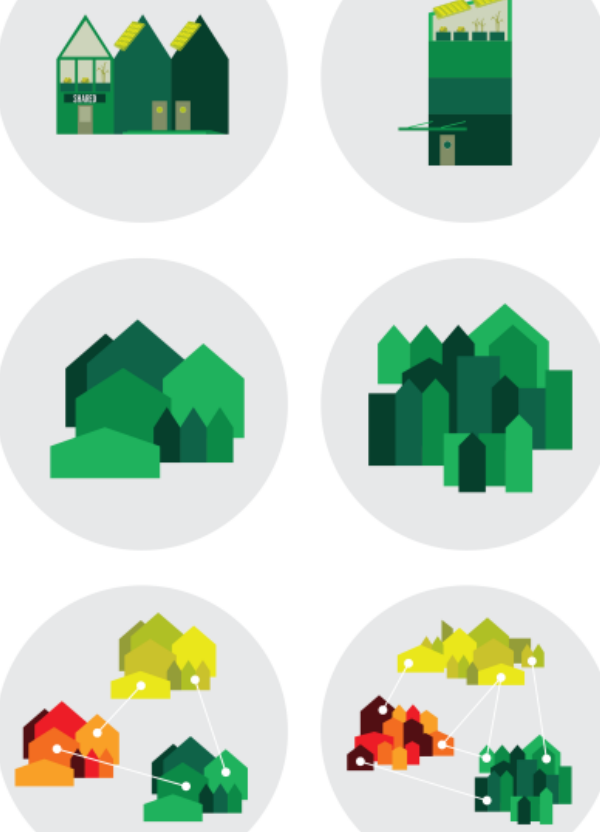

scale of density

'Micro-Village' is a term which refers to the personal or domicile ecosystem, however that sociospatial organization may manifest. This means all living arrangements can be expressed within the Micro-Village framework. To further clarify, socio-spatial organization refers to the relationships within a given parameter of space. The following socio-spatial examples can provide insight into the range of possible organizations, which include but are not limited to: a nuclear family living in a detached house; individuals co-habiting in a shared unit; a self-organized encampment of unhoused individuals; a housing cooperative of like-minded individuals pursuing a specific goal; a co-parenting arrangement between single parents in duet-style townhouses; a re-entry facility for formerly incarcerated individuals; or a needs-based care facility for the elderly.

'Macro-Village' refers to the community/neighborhood/city/regional scale. Examples of MacroVillages include school districts; sanitation districts; ecological watershed regions; or a city, town or neighborhood depending on scale of population density.

'Multi-Village' refers to the co-ordination between Macro-Villages; a network of Macro-Villages; specifically the communication and coordination of infrastructure between them, Examples of MultiVillage networks include: transportation infrastructure; communication infrastructure; mechanisms to regulate exploitation; as well as knowledge and learning infrastructure, among others. 


\subsection{Proposal: Visual framing to facilitate comprehension and communication}

As this article is part of a larger body of ongoing research, the following diagram represents the inprocess development of a new visual framing of the 'ecosystem of needs' at the Micro-Village scale. The goal of diagrammatic development is to help facilitate assessment of one's own personal ecosystem, and after analysis, help guide appropriate action-planning to build POP and empower wellbeing. An extensive literature review of academic studies and examination of primary and secondary sources has been conducted in order to establish the following categories of priority within the MicroVillage ecosystem. The following diagram has been derived from this research in the attempt to establish a visual framework for ecosystemic, socio-spatial resilience at the Micro-Village scale.

Figure 8. Image created by author.

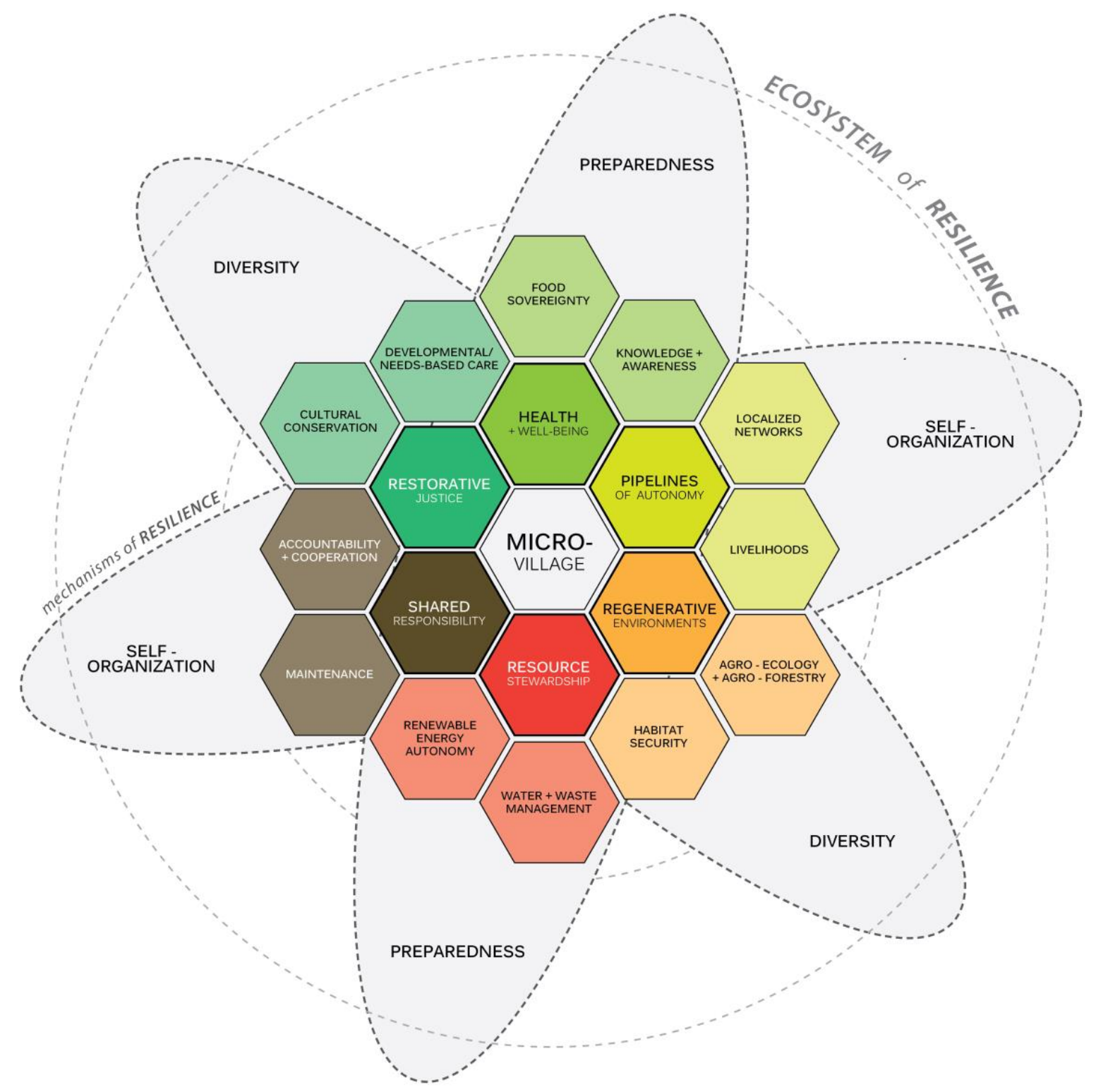


Figures 9-11. Images created by author.
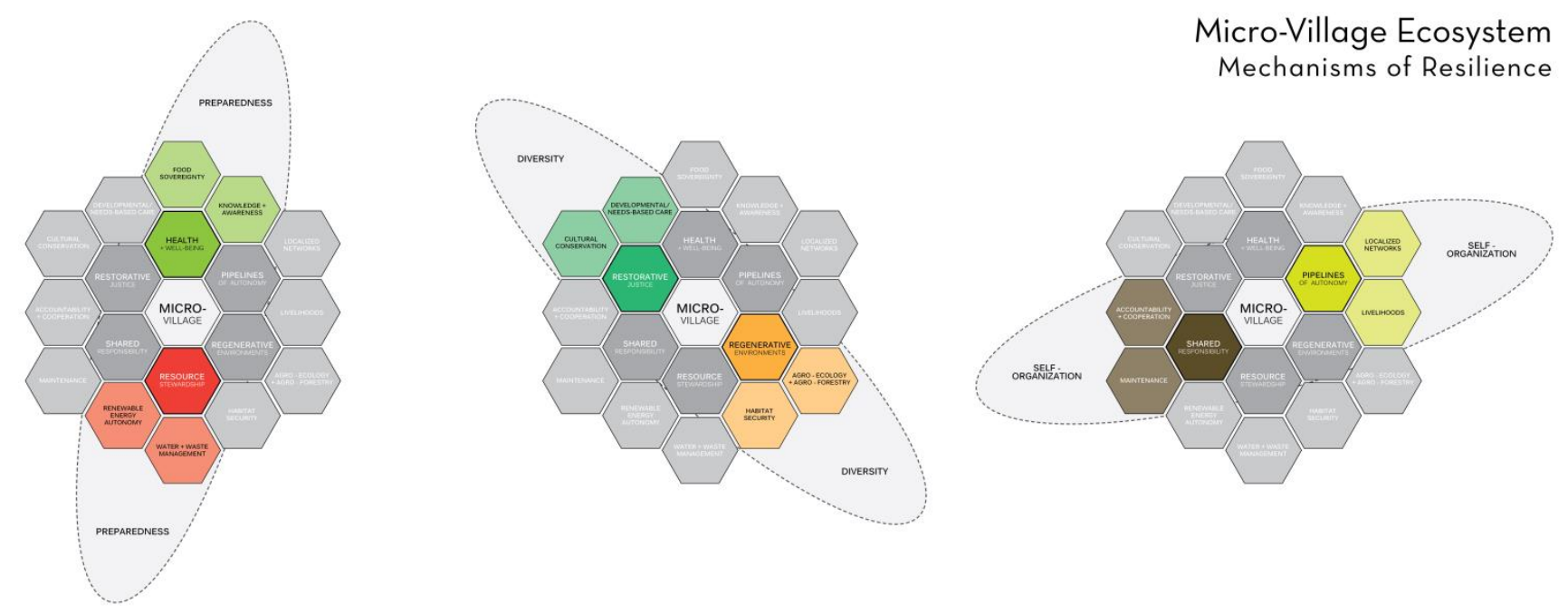

The diagrammatic sequence above endeavors to highlight how socio-spatial environments function as ecosystems, specifically through three interdependent mechanisms of resilience which empower POP.

Similarly, the chart below includes much of the same information, represented in a different manner.

Figure 12. Image created by author.

\begin{tabular}{|c|c|c|}
\hline Resilience Mechanism & Aspect & Need \\
\hline \multirow[t]{4}{*}{ Preparedness } & Resource Stewardship & Water + Waste Management \\
\hline & & Renewable Energy Autonomy \\
\hline & Health + Well-being & Food Sovereignty \\
\hline & & Knowledge + Awareness \\
\hline \multirow{4}{*}{ Diversity } & Restorative Justice & Developmental / Needs-Based Care \\
\hline & & Cultural Conservation \\
\hline & Regenerative Environments & Agro-Ecology + Agro-Forestry \\
\hline & & Habitat Security \\
\hline \multirow[t]{4}{*}{ Self-Organization } & Shared Responsibility & Accountability + Cooperation \\
\hline & & Maintenance \\
\hline & Pipelines of Autonomy & Livelihoods \\
\hline & & Localized Networks \\
\hline
\end{tabular}

\section{$\underline{\text { Understanding Mechanisms of Resilience within Micro-Village ecosystems }}$}

Micro-Village resilience necessitates 'preparedness'- or needs fulfillment- which includes access to food and energy, and considers both waste and water management. These aspects correspond to health 
for equitable well-being, and resource stewardship - a necessary consideration in ecological stability. Preparedness subsequently relates to the second resilience mechanism of diversity, specifically through agro-ecology in facilitating food access, as well as in protecting environments, in particular, potable water sources.

Micro-Village resilience should also empower 'diversity'- or equity, which includes aspects of both restorative justice, and an emphasis on regenerative environments. This is of crucial consideration because this mechanism reflects the understanding of the reality of how people experience life differently - they come from different cultures, all of which deserve respect, and experience a range of physiological and psychological conditions, which are person-specific. Similarly, habitat security refers to the need and corresponding right of all life to stability and security. For humans this may translate to current concepts of land rights and tenure, yet in general for all living species, it refers to the ecological stability necessary within their contextual environments for ongoing well-being, as well as protection from threats posed by an external hierarchy. From an ecological perspective, this security is closely linked with the tactics of Agro-Ecology and Agro-Forestry, which the international peasant's movement and indigenous leaders have determined are critical for successful stewardship of land management and environmental well-being ${ }^{8}$.

The final mechanism of Micro-Village resilience is 'self-organization', or sovereignty. This mechanism specifically recognizes the accountability, cooperation, and trust necessary for equitable, shared responsibility. A pipeline of autonomy makes the most of the lateral trust developed through equitable, shared responsibility, and helps create a network of support between Micro-Villages. This network facilitates equitable growth through the tactic of cooperative, restorative economics. Nwamaka Agbo describes the methodology of restorative economics as one which "leverages community-owned and community-governed projects to bring residents together to create shared prosperity and self-determination and in turn build collective power"’.

By localizing the fulfillment of needs within parameters of ecological stability, regions will become increasingly self-sufficient. The consequences of climate change as outlined in the IPCC report will require context-specific action-planning, and this autonomy can provide regions with the flexibility to

\footnotetext{
${ }^{8}$ The follow excerpt is from a declaration read aloud by Mariam Sou, an agroecological peasant farmer from ENDA-Tiers Monde, Senegal, representing La Via Campesina at the II International Symposium on Agroecology in April of 2018:
}

“Agroecology cannot be understood as a simple set of techniques and productive practices.

Agroecology is a way of life of our peoples, in harmony with the language of Nature. It is a paradigm shift in the social, political, productive and economic relations in our territories, to transform the way we produce and consume food and to restore a socio-cultural reality devastated by industrial food production. Agroecology generates local knowledge, builds social justice, promotes identity and culture and strengthens the economic viability of rural and urban areas.

This vision, and these principles and common values of Agroecology, agreed upon at the International Forum on Agroecology in Nyéléni in 2015, have been continuously enriched, innovated, adapted, multiplied, implemented in different ways according to our varied realities and respecting our worldviews, cultures, economies and local food systems, based on the real production of agroecological food."

${ }^{9}$ Nwamaka Agbo. Accessed at: https://www.nwamakaagbo.com/restorative-economics/ 
respond to challenges that arise with specificity. Furthermore, this localization of needs fulfillment and thus governance increases the likelihood of legitimately democratic control over the systems which affects individual lives.

While self-determination is touted as a principle of the growth-prioritized model, the diverse reality of lived experiences demonstrates that there is hierarchical power and systemic privilege in access within-in the socio-spatial infrastructure of capitalism. As societal inequity has been demonstrated to be intrinsic to the growth-based model, bottom-up decision-making control must be prioritized to subvert the existing hierarchical power dynamic.

\section{Next Steps}

As this article is written in the preliminary stage of a larger body of doctoral investigation, this section endeavors to explain how this research intends to proceed.

\subsection{Linguistic and Visual Framing of the Ecosystemic model: Continued Development}

This research aims to facilitate individual understanding of one's own personal operating power [POP]. Subsequently, comprehension is predicated on an individual internalizing the ecosystemic nature of needs, and how that correlates to one's own experience and context. To this end, this research intends to develop a glossary to support the linguistic framing of the ecosystemic model, which can be used to propagate assessment, analysis and action-planning that facilitates societal equity and ecological stability. Furthermore, corresponding visual framing will be developed to additionally facilitate successful comprehension through diagrammatic representation.

\subsection{Developing a Participatory Process: Making Theory Useful for Individuals and Communities}

Development of a participatory process will be crucial for translating theory to practice (Hamdi 2010). In order to reach critical mass of societal acceptance, said process should be presented in a flexible manner to facilitate widespread and diverse access to bottom-up assessment. Yet the usefulness of such a process will be dependent on successful communication, which requires specific attention to linguistic and visual framing. If successfully internalized, subsequent peer-to-peer, lateral sharing of the participatory process can further facilitate the normalization of ecosystemic framing.

Figure 13. Image created by author.

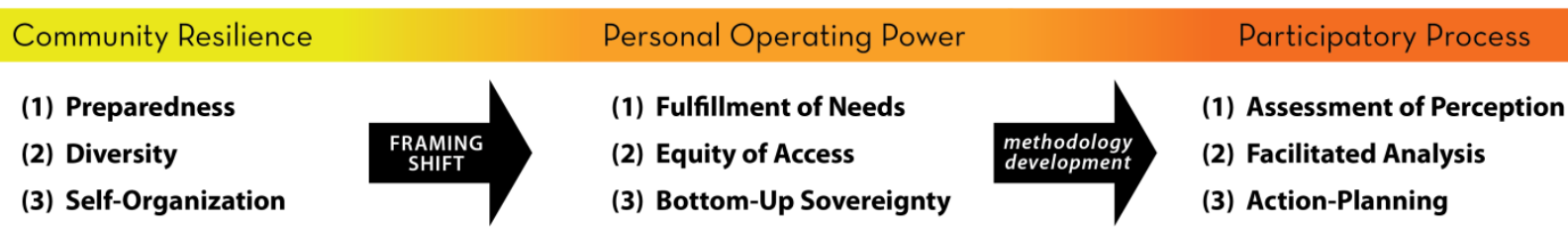

This research subsequently intends to test the developed participatory process through a series of semi-structured interviews, specifically with community organizers, who function as conduits of knowledge and facilitators of interpersonal connection and action-planning. 


\section{Conclusions}

The growth-based paradigm which governs societal organization and infrastructure has been conclusively demonstrated by the 2018 IPCC report, amongst a litany of evidences, to be intrinsically incapable of ensuring ecological stability and societal equity. With this understanding, humanity must re-evaluate the priorities which have been embedded within our cultural psychologies; priorities which reinforce the growth-based paradigm through intentional linguistic framing. In the effort to promote this vital shift towards an ecosystemic model of living, this body of research endeavors to facilitate comprehension and communication, through the development of linguistic and visual framing tools.

In the effort to facilitate a paradigm which can empower societal equity and ecological stability, the following takeaways are crucial prerequisite understandings to the future development of this research:

i. Linguistic and visual framing are powerful tools that influence comprehension. Language choice, as well as graphic representation must be paid close attention, to facilitate internalization of the needed ecosystemic paradigm.

ii. Rather than as a 'hierarchy of needs', needs must be understood as an ecosystem of interdependent variables. This 'ecosystem of needs' includes the internal factors of physiological needs, psychological needs, as well as the external variables of interpersonal needs, and environmental needs, both built and natural.

iii. Resilience at the individual scale should be understood as 'Personal Operating Power' [POP]. The successful facilitation of POP can be understood by the following attributes:

1. Fulfillment of the Ecosystem of Needs

2. Equity of Access

3. Bottom-up Sovereignty

iv. To ensure equitable resilience, resilience must be examined from the bottom-up; prioritizing localization of needs fulfillment. In socio-spatial terms, this means assessment, analysis, and action-planning at the personal, or domicile scale, which in ecosystemic framing would be termed the 'Micro-Village'.

v. The linguistic framing of socio-spatial infrastructure as multi-scalar ecosystems is imperative to achieving ecological stability and societal equity. The linguistic framing of 'Micro-Villages', 'Macro-Villages', and 'Multi-Villages' can provide a communication framework to facilitating comprehension and promote a shift towards ecosystemic action-planning, and ultimately — societal well-being. 


\section{References}

Anguelovski, Isabelle. "Alternative Food Provision Conflicts in Cities: Contesting Food Privilege, Injustice, and Whiteness in Jamaica Plain, Boston." Geoforum 58 (2015): 184-94. doi:10.1016/j.geoforum.2014.10.014.

Baradaran, Mehrsa. The Color of Money: Black Banks and the Racial Wealth Gap. Cambridge, MA: Belknap Press of Harvard University Press, 2017.

BondGraham, Darwin. "United Nations Expert Describes Oakland and California's Homeless Crisis as 'Cruel'." East Bay Express. January 21, 2018. Accessed May 11, 2018. https://www.eastbayexpress.com/SevenDays/archives/2018/01/21/united-nations-expertdescribes-oakland-and-californias-homeless-crisis-as-cruel.

Brones, Anna. "Food Apartheid: The Root of the Problem with America's Groceries." The Guardian. May 15, 2018. Accessed June 3, 2018. https://www.theguardian.com/society/2018/may/15/foodapartheid-food-deserts-racism-inequality-america-karen-washington-interview.

Chomsky, Noam. Power Systems: Conversations with David Barsamian on Global Democratic Uprisings and the New Challenges to U.S. Empire. London: Penguin Books, 2013.

Gee, Alastair. "America's Homeless Population Rises for the First Time since the Great Recession." The Guardian. December 06, 2017. Accessed October 19, 2018. https://www.theguardian.com/usnews/2017/dec/05/america-homeless-population-2017-official-count-crisis.

Hamdi, Nabeel. The Placemaker's Guide to Building Community. London: Earthscan, 2010.

Hickel, Jason. "Why Growth Can't Be Green." Foreign Policy. September 12, 2018. Accessed September 14, 2018. https://foreignpolicy.com/2018/09/12/why-growth-cant-be-green/.

Housing \& Dignity Project. Housing Oakland's Unhoused. Oakland, CA, 2018.

IPCC. Global Warming of 1.5 degrees Celsius. Switzerland, 2018.

Klein, Naomi. "A Radical Vatican?" The New Yorker. July 10, 2015. Accessed September 1, 2018. https://www.newyorker.com/news/news-desk/a-visit-to-the-vatican.

Klein, Naomi. The Battle for Paradise: Puerto Rico Takes on the Disaster Capitalists. Chicago, IL: Haymarket Books, 2018.

Lakoff, George. 1996. Moral Politics: How Liberals and Conservatives Think. $1^{\text {st }}$ ed. Chicago: University of Chicago Press, ISBN 978-0-226-46805-1. 
Lakoff, George. 2004. Don't Think of an Elephant! Know Your Values and Frame the Debate.

Vermont: Chelsea Green Publishing Company, ISBN 1-931498-71-7.

Letters. "The EU Needs a Stability and Wellbeing Pact, Not More Growth." The Guardian. September 16, 2018. Accessed September 16, 2018. https://www.theguardian.com/politics/2018/sep/16/theeu-needs-a-stability-and-wellbeing-pact-not-more-growth.

McSweeney, Robert, and Rosamund Pearce. "Interactive: The Impacts of Climate Change at 1.5C, $2 \mathrm{C}$ and beyond." Interactive: The Impacts of Climate Change at $1.5 \mathrm{C}, 2 \mathrm{C}$ and beyond | Carbon Brief. Accessed September 14, 2018. https://interactive.carbonbrief.org/impacts-climatechange-one-point-five-degrees-two-degrees/.

Mease, Lindley. "Rosalinda Guillen: Movement-Building for Farmworker Justice." Medium. April 01, 2018. Accessed September 26, 2018. https://medium.com/blue-heart/rosalinda-guillen-movementbuilding-for-farmworker-justice-8c507fefe $8 \mathrm{cb}$.

NOAA. Patterns and Projections of High Tide Flooding along the U.S. Coastline Using a Common Impact Threshold. Silver Spring, MD, 2018.

O’Neill, Daniel W., Andrew L. Fanning, William F. Lamb, and Julia K. Steinberger. "A Good Life for All within Planetary Boundaries." Nature Sustainability 1, no. 2 (2018): 88-95. doi:10.1038/s41893-018-0021-4.

Samuel, Leah. "Not Just Pirates Anymore: Scurvy Afflicts the Poor and Homeless." STAT. November 30, 2015. Accessed August 22, 2018. https://www.statnews.com/2015/11/27/scurvy/.

Schipper, E. Lisa F. and Lara Langston. A comparative overview of resilience measurement frameworks. London, Overseas Development Institute, 2015.

Stiglitz, Joseph E. Globalization and Its Discontents. England: Penguin, 2002.

Verburg, Peter H., Ole Mertz, Karl-Heinz Erb, Helmut Haberl, and Wenbin Wu. "Land System Change and Food Security: Towards Multi-scale Land System Solutions." Current Opinion in Environmental Sustainability 5, no. 5 (2013): 494-502. doi:10.1016/j.cosust.2013.07.003.

Wallace-Wells, David. "UN Says Climate Genocide Is Coming. It's Actually Worse Than That." Daily Intelligencer. October 10, 2018. Accessed October 11, 2018. http://nymag.com/intelligencer/2018/10/un-says-climate-genocide-coming-but-its-worse-thanthat.html. 


\section{Acknowledgments}

I would like to thank Nicholas Tomaszewski and Madison Pauly for the editorial feedback and insights they provided, as well as acknowledge my doctoral advisors, Dr. Felipe Pich-Aguilera and Dr. Jordi Rovira at the Universitat Internacional de Catalunya (UIC).

\section{Conflict of Interest}

The author, Jessica Rose Pauly, receives funding to support doctoral research from the Generalitat de Catalunya, Department de Treball, Afers Socials I Families. Exp. 080620180001779.

(C) 2018 by the authors; licensee MDPI and IFoU, This article is an open access article distributed under the terms and conditions of the Creative Commons Attribution license. 